\title{
Assessment of Quality of Life and Sexual Function in ladies with Pregnancy-Related Skin Changes
}

\author{
Hesham Nabil Khalid El Shamy, Samar Magdy Abdelhady Hassan*, Heba Allah saad Eldeen Bazid \\ Department of Dermatology, Andrology \& STDs, Faculty of Medicine, Menoufia University, Menoufia, Egypt \\ *Corresponding Author: Samar Magdy Abdelhady Hassan, Mobile: (+20) 01127045558,
}

E-mail: moramagdy93@gmail.com

\begin{abstract}
Background: Pregnancy is characterized by immunologic, metabolic, endocrine, and vascular changes, which lead to skin changes, making pregnant women suffer from body image disorder, physical performance, and mental health affection which adversely affect their sexual activity and quality of life.

Objectives: To evaluate the impact of pregnancy and pregnancy-related changes on the quality of life and sexual function.

Patients and Methods: This case-control study involved 160 females divided into 4 groups: 40 pregnant females in the $1^{\text {st }}$ trimester, 40 in the $2^{\text {nd }}$ trimester, 40 in the $3^{\text {rd }}$ trimester, and 40 nonpregnant females as the control group. Every participant filled out the Arabic version of the Female Sexual Dysfunction Index (FSDI) and Dermatology Life Quality Index (DLQI).

Results: There was a significant difference between the three trimesters regarding the types of skin changes $(\mathrm{p}=0.006)$. Acne vulgaris was the most common change in the $1^{\text {st }}$-trimester group. There was a significant difference between the studied groups as regards the female sexual function ( $<<0.001$ regarding FSDI total score). There was no significant difference between females with skin changes compared to those without regard to their sexual function during $1^{\text {st }}, 2^{\text {nd }}$, and $3^{\text {rd }}$ trimesters $(\mathrm{P}=0.07,0.5,0.6$ resp) regarding FSDI total score. Regarding the DLQI score, there was a significant difference between both the $2^{\text {nd }}$ and $3^{\text {rd }}$ trimesters and control groups $(\mathrm{P} 2=0.01$ and $\mathrm{P} 3=0.009$ respectively) with higher mean values of the DLQI score among females with skin changes.

Conclusion: Pregnancy negatively affected female sexual function and quality of life. Skin changes during pregnancy significantly affected the quality of life of pregnant females.
\end{abstract}

Keywords: Female sexual function, Pregnancy, Quality of life.

\section{INTRODUCTION}

Pregnancy is a physiological state characterized by profound immunologic, metabolic, endocrine, and vascular changes, which make the pregnant woman susceptible to changes in the skin and its appendages. These alterations may range from normal cutaneous changes that occur with almost all pregnancies to common diseases that are not associated with pregnancy, to eruptions that appear to be specifically associated with pregnancy ${ }^{(1)}$.

Physiological skin conditions including striaegravidarum and hyperpigmentation and nails, hair, vascular, and glandular changes. Many of these alterations regress significantly within the 1-6 months postpartum (1). Unpleasant skin changes including acne, melasma, and striae make pregnant women suffer from body image disorder, leading to their decreased self-esteem and feeling ugly affecting their sexual response ${ }^{(2)}$.

During pregnancy, organic and hormonal variations change the physical performance and mental health of women ${ }^{(3)}$. These normal changes and their interactions with certain factors (e.g., economic factors and stressful events) may adversely affect women's health throughout the stages of pregnancy even if they do not have any medical or psychological disease. This consequently affects women's usual activities and quality of life ${ }^{(4)}$.

The work aimed to evaluate the impact of pregnancy and pregnancy-related changes on the quality of life and sexual function.

\section{PATIENTS AND METHODS}

This case-control study was conducted on a total number of 160 women: 40 pregnant females in the first trimester, 40 pregnant females in the second trimester, 40 pregnant females in the third trimester, and 40 nonpregnant females as controls. Each trimester was divided into two subgroups: women with pregnancyrelated skin changes and women without any pregnancy-related skin changes. Cases are collected from, Dermatology outpatients clinic Menoufia University hospital.

After ethical committee approval, we take formal consent from every participant. A complete history of the patients was taken including age, education level, residence, and occupation; marital history including duration of the marriage, parity, age, education, and special habits as smoking of the husband; present history including type, onset, course, duration of the skin changes, relation to the number of pregnancy, family history and the presence of associated 
comorbidities such as cardiovascular disease, diabetes mellitus, and thyroid diseases.

\section{Ethical considerations:}

An approval of the study was obtained from Menoufia University academic and ethical committee. Every patient signed an informed written consent for acceptance of the operation.

Any female who had any of the following was excluded: other skin diseases and associated comorbidities such as cardiovascular disease, diabetes mellitus, and thyroid diseases, females with any gynecological or obstetric problems or take any drug that may affect their quality of life and sexual function, and females whom their husbands had any type of sexual dysfunction.

The sexual function of participating women was assessed by using the Arabic version of the Female Sexual Function Index (FSDI) Questionnaire which was prepared using the back-translation technique ${ }^{(5)}$, and quality of life was evaluated by the Dermatology Life Quality Index (DLQI) ${ }^{(6)}$.

\section{Statistical analysis}

Results were collected, tabulated, and statistically analyzed by (IBM SPSS Statistics for Windows, Version 23.0. Armonk, NY: IBM Corp.).Two types of statistical analyses were conducted: descriptive statistics, for example, number (No), percentage (\%) mean $(\overline{\mathrm{x}})$, standard deviation (SD), and median and range; analytic statistics, for example, Student's $t$-test is a test of significance used for comparison of quantitative variables between two groups of normally distributed data, ANOVA test was used for comparison of quantitative variables between more than two groups of normally distributed data with multiple comparisons, and Chi-squared test $\left(\chi^{2}\right)$ was used to study the association between qualitative variables and $\mathrm{P}$-value of $<0.05$ was considered statistically significant.

\section{RESULTS}

There was no significant difference between the studied groups regarding sociodemographic data $(\mathrm{p}<0.05)$. (Table 1)

Table (1): Socio-demographic data of the studied groups

\begin{tabular}{|c|c|c|c|c|c|c|c|}
\hline Variables & $\begin{array}{c}\mathbf{1}^{\text {st }} \text { trimester } \\
\text { pregnant } \\
(\mathrm{n}=40) \\
\text { No }(\%)\end{array}$ & $\begin{array}{c}2^{\text {nd }} \text { trimester } \\
\text { pregnant } \\
(\mathrm{n}=40) \\
\text { No }(\%)\end{array}$ & $\begin{array}{c}\text { 3rd trimester } \\
\text { pregnant } \\
(\mathrm{n}=40) \\
\text { No }(\%)\end{array}$ & $\begin{array}{c}\text { Control } \\
\text { group } \\
(\mathrm{n}=40) \\
\text { No }(\%)\end{array}$ & P1 & $\mathbf{P 2}$ & P3 \\
\hline $\begin{array}{l}\text { Age (years): } \\
16-<25 \\
25-<35 \\
35-45 \\
\text { Mean } \pm \text { SD } \\
\text { range }\end{array}$ & $\begin{array}{c}14(35.0) \\
21(52.5) \\
5(12.5) \\
27.25 \pm 5.40 \\
16-38 \\
\end{array}$ & $\begin{array}{c}10(25.0) \\
27(67.5) \\
3(7.5) \\
28.05 \pm 4.79 \\
19-38\end{array}$ & $\begin{array}{c}14(35.0) \\
21(52.5) \\
5(12.5) \\
27.25 \pm 5.20 \\
18-40\end{array}$ & $\begin{array}{c}12(30.0) \\
21(52.5) \\
7(17.5) \\
28.08 \pm 5.01 \\
19-42\end{array}$ & 0.784 & 0.282 & 0.784 \\
\hline $\begin{array}{l}\text { Residence: } \\
\text { Rural } \\
\text { Urban }\end{array}$ & $\begin{array}{c}5(12.5) \\
35(87.5)\end{array}$ & $\begin{array}{l}11(27.5) \\
29(72.5)\end{array}$ & $\begin{array}{l}12(30.0) \\
28(70.0)\end{array}$ & $\begin{array}{l}11(27.5) \\
29(72.5)\end{array}$ & 0.094 & NA & 0.805 \\
\hline $\begin{array}{l}\text { Education levels: } \\
\text { Illiterate } \\
\text { Read \& write } \\
\text { Secondary } \\
\text { education } \\
\text { University\& above } \\
\end{array}$ & $\begin{array}{c}1(2.5) \\
3(7.5) \\
23(57.5) \\
13(32.5)\end{array}$ & $\begin{array}{c}2(5.0) \\
2(5.0) \\
18(45.0) \\
18(45.0)\end{array}$ & $\begin{array}{c}3(7.5) \\
2(5.0) \\
17(42.5) \\
18(45.0)\end{array}$ & $\begin{array}{c}0 \\
1(2.5) \\
27(67.5) \\
12(30.0)\end{array}$ & 0.501 & 0.149 & 0.078 \\
\hline $\begin{array}{l}\text { Occupation: } \\
\text { Not-working } \\
\text { Working }\end{array}$ & $\begin{array}{c}31(77.5) \\
9(22.5)\end{array}$ & $\begin{array}{c}32(80.0) \\
8(20.0)\end{array}$ & $\begin{array}{l}30(75.0) \\
10(22.0)\end{array}$ & $\begin{array}{l}27(67.5) \\
13(32.5)\end{array}$ & 0.317 & 0.204 & 0.459 \\
\hline
\end{tabular}

$\mathrm{P} 1: 1^{\text {st }}$ trimester vs controls P2: $2^{\text {nd }}$ trimester vs controls P3: 3rd trimester vs controls

There was a significant difference between pregnant females in the three trimesters' groups regarding the types of skin changes $(\mathrm{p}=0.006)$. Acne vulgaris was the most common change in the $1^{\text {st }}$-trimester group, while generalized hyperpigmentation was common among the $2^{\text {nd }}$ and the $3^{\text {rd }}$ trimesters' groups. There was a significant relation regarding the skin changes and the number of previous pregnancies as skin changes appeared in 14 cases of the firsttrimester group who had previous pregnancies and in 5 cases of $2^{\text {nd }}$ trimester and 8 cases of $3^{\text {rd }}$-trimester groups $(\mathrm{p}=0.014)$. There was a highly significant difference between the three trimesters regarding the duration of skin changes during pregnancy $(\mathrm{p}<0.001)$, with the longest duration in the $3^{\text {rd }}$ trimester and shortest in the $1^{\text {st }}$ trimester.

(Table 2) 
Table (2): Comparison between pregnant females at different trimesters regarding the present history of pregnancy-related skin changes.

\begin{tabular}{|c|c|c|c|c|c|c|c|c|}
\hline \multirow{2}{*}{ Variables } & \multicolumn{2}{|c|}{$\begin{array}{c}\mathbf{1}^{\text {st }} \text { trimester } \\
(\text { No }=\mathbf{2 0})\end{array}$} & \multicolumn{2}{|c|}{$\begin{array}{c}2^{\text {nd }} \text { trimister } \\
(\text { No }=20)\end{array}$} & \multicolumn{2}{|c|}{$\begin{array}{c}3^{\text {rd }} \text { trimister } \\
(\text { No }=20)\end{array}$} & \multirow[t]{2}{*}{$\chi^{2}$} & \multirow{2}{*}{ p-value } \\
\hline & No & $\%$ & No & $\%$ & No & $\%$ & & \\
\hline $\begin{array}{ll}\text { Types: } \\
\text { - } & \text { Acne vulgaris } \\
\text { - } & \text { Axillary and genital } \\
& \text { hyperpigmentation } \\
\text { - } & \text { Linea nigra } \\
\text { - } & \text { Melasma } \\
\text { - } & \text { Secondary areola } \\
\text { - } & \text { Striagravidarum } \\
\text { - } & \text { Generalized } \\
& \text { hyperpigmentation } \\
\text { - } & \text { Varicose veins } \\
\end{array}$ & $\begin{array}{c}10 \\
3 \\
1 \\
1 \\
3 \\
2 \\
0 \\
0 \\
\end{array}$ & $\begin{array}{c}50 \\
15 \\
\\
5 \\
5 \\
15 \\
10 \\
0 \\
\\
0 \\
\end{array}$ & $\begin{array}{l}2 \\
2 \\
2 \\
3 \\
0 \\
5 \\
5 \\
1 \\
\end{array}$ & $\begin{array}{c}10 \\
10 \\
10 \\
15 \\
0 \\
25 \\
25 \\
5 \\
\end{array}$ & $\begin{array}{l}2 \\
3 \\
1 \\
1 \\
1 \\
2 \\
6 \\
1 \\
1\end{array}$ & $\begin{array}{c}10 \\
15 \\
\\
5 \\
5 \\
5 \\
10 \\
30 \\
\\
5 \\
\end{array}$ & 10.35 & 0.006 \\
\hline $\begin{array}{ll} & \text { Duration (week): } \\
\text { - } & 1 \\
\text { - } & 2 \\
\text { - } & 3 \\
\text { - } & >3\end{array}$ & $\begin{array}{l}8 \\
5 \\
5 \\
2\end{array}$ & $\begin{array}{l}40 \\
25 \\
25 \\
10\end{array}$ & $\begin{array}{c}1 \\
1 \\
1 \\
17\end{array}$ & $\begin{array}{c}5 \\
5 \\
5 \\
85\end{array}$ & $\begin{array}{c}0 \\
0 \\
1 \\
19\end{array}$ & $\begin{array}{c}0 \\
0 \\
5 \\
95\end{array}$ & 37.87 & $<0.001$ \\
\hline $\begin{array}{l}\text { Relation to the number } \\
\text { of pregnancy: } \\
\text { - } \mathrm{No} \\
\text { - } \quad \text { Yes } \\
\end{array}$ & $\begin{array}{c}6 \\
14 \\
\end{array}$ & $\begin{array}{l}30 \\
70 \\
\end{array}$ & $\begin{array}{c}15 \\
5 \\
\end{array}$ & $\begin{array}{l}75 \\
25 \\
\end{array}$ & $\begin{array}{c}12 \\
8 \\
\end{array}$ & $\begin{array}{l}60 \\
40 \\
\end{array}$ & 8.48 & 0.014 \\
\hline $\begin{array}{ll} & \text { Family history : } \\
\text { - } & \text { Negative } \\
\text { - } & \text { Positive } \\
\end{array}$ & $\begin{array}{l}10 \\
10\end{array}$ & $\begin{array}{l}50 \\
50\end{array}$ & $\begin{array}{l}10 \\
10\end{array}$ & $\begin{array}{l}50 \\
50\end{array}$ & $\begin{array}{c}9 \\
11\end{array}$ & $\begin{array}{l}45 \\
55\end{array}$ & 0.13 & 0.935 \\
\hline
\end{tabular}

No: Number \%: Percent

There was a significant difference between the studied groups regarding the frequency of intercourse $(\mathrm{p}=0.041)$. There was a highly significant difference between the studied groups regarding the FSDI score $(\mathrm{p}<0.001)$. A high significant difference between the studied groups regarding desire score was reported $(\mathrm{p}>0.001)$. There was a significant difference between the studied groups in terms of the lubrication score $(p=0.013)$. There was a significant difference between the studied groups in terms of orgasm score $(\mathrm{p}=0.010)$. There was a highly significant difference between the studied groups regarding pain score $(\mathrm{p}<0.001)$.

There was no significant difference between the 1st-trimester pregnant females and the control group regarding their sexual function. There were significantly lower mean scores of arousal in the 2nd-trimester pregnant females than the control group ( $\mathrm{p} 2=0.020$ ). There was a significantly lower mean score of pain in the 2 nd-trimester pregnant females than the control group ( $\mathrm{p} 2=0.009$ ). There was a significantly lower mean score of FSDI in the 2nd-trimester pregnant females than the control group $(\mathrm{p} 2=0.053)$. There were no significant differences between the 2 nd-trimester group and control regarding the frequency of sexual intercourse, prevalence of female sexual dysfunction, desire, lubrication, orgasm, and satisfaction. There was a significant difference between 3rd-trimester pregnant females and control $(\mathrm{p} 3=0.005)$. Also, the mean values of sexual pain were significantly lower among the 3rd trimester pregnant females than the control group, as the $(\mathrm{p} 3=0.015)$. There was no significant difference between the 3rd-trimester group and control regarding desire, arousal, lubrication, orgasm, and satisfaction. (Table 3) 
Table (3): Sexual functions among the studied groups

\begin{tabular}{|c|c|c|c|c|c|c|c|c|c|}
\hline \multirow[t]{2}{*}{ Variables } & \multicolumn{2}{|r|}{$\begin{array}{c}1^{\text {st }} \\
\text { trimester } \\
\text { pregnant } \\
(\mathrm{No}=40)\end{array}$} & $\begin{array}{c}2^{\text {nd }} \\
\text { trimester } \\
\text { pregnant } \\
(\mathrm{No}=40)\end{array}$ & $\begin{array}{c}3^{\text {rd }} \\
\text { trimester } \\
\text { pregnant } \\
(\mathrm{No}=40)\end{array}$ & $\begin{array}{c}\text { Control } \\
\text { group } \\
(\mathbf{N o}=\mathbf{4 0})\end{array}$ & \multirow[t]{2}{*}{ 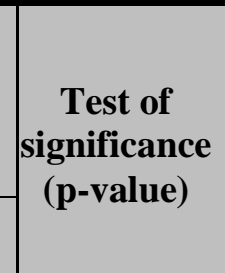 } & \multirow[t]{2}{*}{ P1 } & \multirow[t]{2}{*}{ P2 } & \multirow[t]{2}{*}{$\mathbf{P 3}$} \\
\hline & & $\%$ & $\%$ & No & No & & & & \\
\hline $\begin{array}{ll} & \text { Frequency of } \\
& \text { intercourse: } \\
\text { - } & \text { Once/day } \\
\text { - } & \text { Once/week } \\
\text { - } & \text { Twice/week } \\
\text { - } & 3-4 \text { times/week } \\
\text { - } & \text { Once/month } \\
\text { - } & \text { Twice/month } \\
\end{array}$ & & \begin{tabular}{c|c}
1 & 2.5 \\
11 & 27.5 \\
18 & 45 \\
8 & 20 \\
2 & 5 \\
0 & 0 \\
\end{tabular} & \begin{tabular}{c|c}
0 & 0 \\
9 & 22.5 \\
15 & 37.5 \\
11 & 27.5 \\
3 & 7.5 \\
2 & 5 \\
\end{tabular} & $\begin{array}{c}0 \\
30 \\
55 \\
7.5 \\
7.5 \\
0 \\
\end{array}$ & $\begin{array}{c}0 \\
17.5 \\
42.5 \\
25 \\
0 \\
15 \\
\end{array}$ & $\begin{array}{c}\chi^{2} \\
(\mathbf{0 . 0 4 1})\end{array}$ & 0.071 & 0.247 & 0.005 \\
\hline \multicolumn{10}{|l|}{ FSDIs } \\
\hline $\begin{array}{ll}\text { Desire } \\
\text { - } \quad \text { Mean } \pm \text { SD } \\
\text { - } \quad \text { Median } \\
\end{array}$ & & $\begin{array}{c}3.09 \pm 0.81 \\
3 \\
\end{array}$ & $\begin{array}{c}2.94 \pm 0.86 \\
3 \\
\end{array}$ & $3.06 \pm 0.74$ & \begin{tabular}{|c}
$3.24 \pm 0.98$ \\
3.3 \\
\end{tabular} & $\begin{array}{l}\text { ANOVA } \\
(<\mathbf{0 . 0 0 1})\end{array}$ & 0.473 & 0.150 & 0.371 \\
\hline $\begin{array}{ll}\text { Arousal } \\
\text { - } & \text { Mean } \pm \text { SD } \\
\text { - } & \text { Median } \\
\end{array}$ & & $\begin{array}{c}3.75 \pm 1.05 \\
3.6\end{array}$ & $\begin{array}{c}3.31 \pm 1.23 \\
3.3\end{array}$ & $\begin{array}{c}3.64 \pm 0.77 \\
3.6\end{array}$ & $\begin{array}{c}3.97 \pm 1.25 \\
3.6 \\
\end{array}$ & $\begin{array}{c}\text { ANOVA } \\
(0.066)\end{array}$ & 0.414 & 0.020 & 0.173 \\
\hline $\begin{array}{l}\text { Lubrication } \\
\text { - } \text { Mean } \pm \text { SD } \\
\text { - } \text { Median } \\
\end{array}$ & & $\begin{array}{c}4.50 \pm 0.84 \\
4.7\end{array}$ & $\begin{array}{c}4.67 \pm 0.99 \\
4.5 \\
\end{array}$ & $\begin{array}{c}4.32 \pm 0.82 \\
4.5\end{array}$ & $\begin{array}{c}4.54 \pm 1.25 \\
4.7 \\
\end{array}$ & $\begin{array}{l}\text { ANOVA } \\
(\mathbf{0 . 0 1 3 )}\end{array}$ & 0.866 & 0.615 & 0.350 \\
\hline $\begin{array}{ll}\text { Orgasm } \\
\text { - } & \text { Mean } \pm \text { SD } \\
\text { - } & \text { Median } \\
\end{array}$ & & $\begin{array}{c}4.18 \pm 1.06 \\
4.2 \\
\end{array}$ & \begin{tabular}{|c}
$3.78 \pm 1.31$ \\
3.8 \\
\end{tabular} & $\begin{array}{c}3.72 \pm 1.26 \\
4\end{array}$ & \begin{tabular}{|c}
$4.22 \pm 1.18$ \\
4.4 \\
\end{tabular} & $\begin{array}{c}\text { ANOVA } \\
(\mathbf{0 . 0 1 0})\end{array}$ & 0.866 & 0.118 & 0.068 \\
\hline $\begin{array}{ll}\text { Satisfaction } \\
\text { - } & \text { Mean } \pm \text { SD } \\
\text { - } & \text { Median } \\
\end{array}$ & & $\begin{array}{c}4.80 \pm 1.11 \\
4.8 \\
\end{array}$ & $\begin{array}{c}4.29 \pm 1.15 \\
4.4 \\
\end{array}$ & $\begin{array}{c}4.53 \pm 1.13 \\
4.8 \\
\end{array}$ & \begin{tabular}{|c|}
$4.64 \pm 1.42$ \\
4.8 \\
\end{tabular} & $\begin{array}{c}\text { ANOVA } \\
(0.101) \\
\end{array}$ & 0.576 & 0.229 & 0.702 \\
\hline $\begin{array}{ll}\text { Pain } \\
\text { - } & \text { Mean } \pm \text { SD } \\
\text { - } & \text { Median } \\
\end{array}$ & & $\begin{array}{c}3.67 \pm 1.06 \\
3.6 \\
\end{array}$ & $\begin{array}{c}3.30 \pm 1.20 \\
3.6 \\
\end{array}$ & $\begin{array}{c}3.40 \pm 1.02 \\
3.2 \\
\end{array}$ & $\begin{array}{c}4.00 \pm 1.15 \\
4 \\
\end{array}$ & 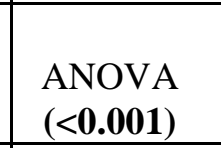 & 0.32 & 0.009 & 0.015 \\
\hline $\begin{array}{ll} & \text { Total FSDI score } \\
- & \text { Mean } \pm \text { SD } \\
- & \text { Median } \\
\end{array}$ & & $\begin{array}{c}3.90 \pm 3.86 \\
24.2\end{array}$ & $\begin{array}{c}22.23 \pm 5.36 \\
22.8\end{array}$ & $\begin{array}{c}22.62 \pm 4.15 \\
22.7\end{array}$ & \begin{tabular}{|c|}
$24.63 \pm 5.55$ \\
24.7
\end{tabular} & $\begin{array}{l}\text { ANOVA } \\
(<\mathbf{0 . 0 0 1 )}\end{array}$ & 0.69 & 0.053 & 0.069 \\
\hline
\end{tabular}

P1: $1^{\text {st }}$ trimester vs controls P2: $2^{\text {nd }}$ trimester vs controls P3: 3rd trimester vs controls

No significant differences between females with skin changes compared to those without in terms of their sexual function among the three trimesters were observed. (Table 4). 
Table (4): Sexual functions among the three trimesters pregnant females stratified according to skin changes.

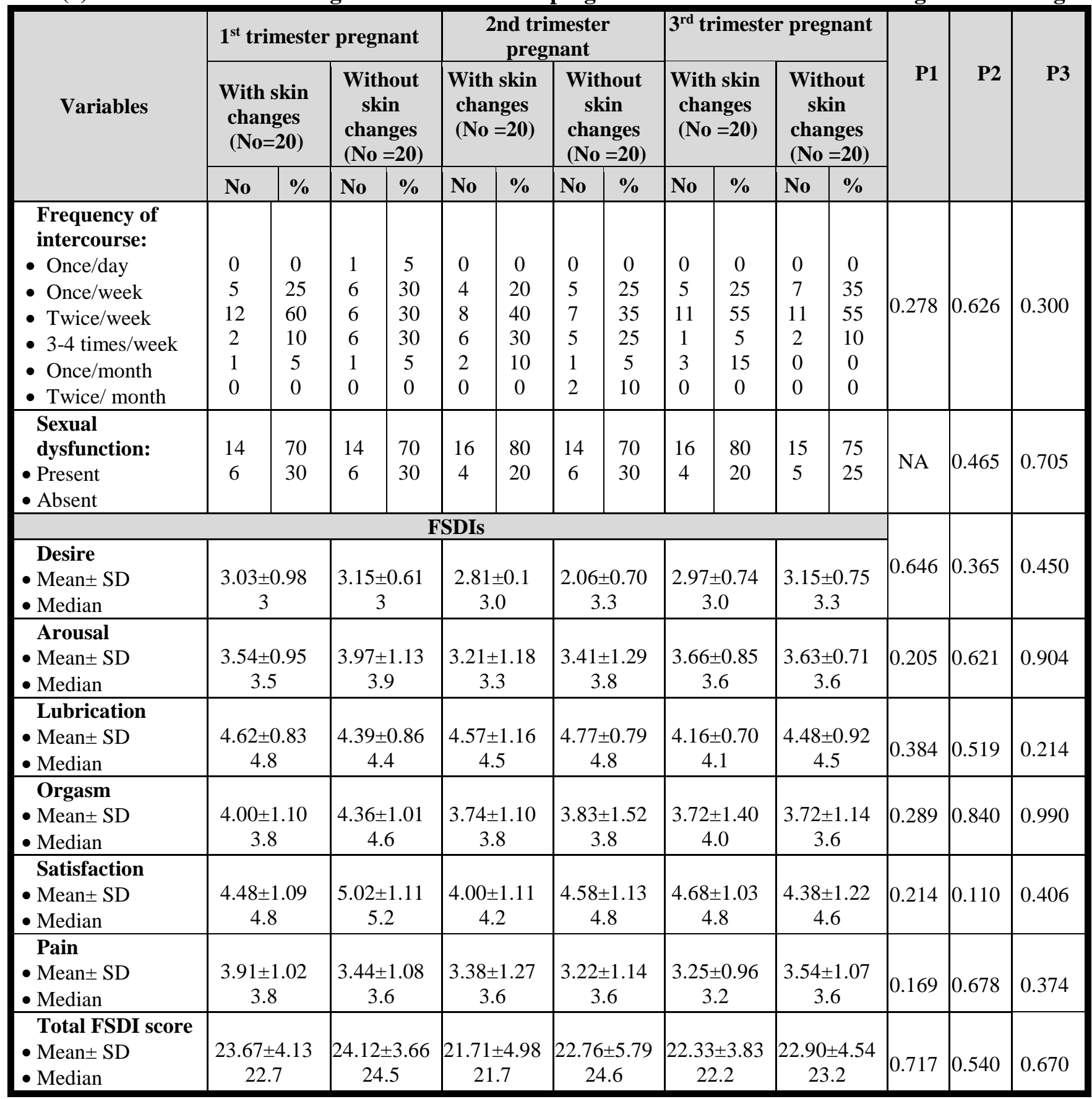

P1: females with skin changes vs females without skin changes in $1^{\text {st }}$ trimester group P2: females with skin changes vs females without skin changes in $2^{\text {nd }}$ trimester group P3: females with skin changes vs females without skin changes in $3^{\text {rd }}$ trimester group There was a significant difference regarding the total score of DLQI between both $2^{\text {nd }}$ and $3^{\text {rd }}$ trimesters and control (means $=4.15,4.1$, and 1.43, respectively) (p-value: $\mathrm{p} 2=0.010$ and $\mathrm{p} 3=0.009$, resp). (Table 5). 
Table (5): DLQI among the studied groups

\begin{tabular}{|c|c|c|c|c|c|c|c|c|c|c|c|c|}
\hline \multirow[t]{2}{*}{ DLQI } & \multicolumn{2}{|c|}{$\begin{array}{c}1^{\text {st }} \text { trimester } \\
\text { pregnant } \\
(\text { No }=40)\end{array}$} & \multicolumn{2}{|c|}{$\begin{array}{c}2^{\text {nd }} \\
\text { trimester } \\
\text { pregnant } \\
(\text { No }=40) \\
\end{array}$} & \multicolumn{2}{|c|}{$\begin{array}{c}3^{\text {rd }} \\
\text { trimester } \\
\text { pregnant } \\
(\text { No }=40) \\
\end{array}$} & \multicolumn{2}{|c|}{$\begin{array}{c}\text { Control } \\
\text { group } \\
\text { No }=40)\end{array}$} & \multirow[t]{2}{*}{$\begin{array}{c}\text { Test of } \\
\text { significance }\end{array}$} & \multirow[t]{2}{*}{ P1 } & \multirow[t]{2}{*}{$\mathbf{P 2}$} & \multirow[t]{2}{*}{ P3 } \\
\hline & No & $\%$ & No & $\%$ & No & $\%$ & No & $\%$ & & & & \\
\hline $\begin{array}{l}\text { No effect at all on } \\
\text { the patient's life }\end{array}$ & 23 & 57.5 & 22 & 55 & 26 & 65.0 & 29 & 72.5 & \multirow{4}{*}{$\chi^{2}$} & \multirow{4}{*}{0.045} & \multirow{4}{*}{0.005} & \multirow{4}{*}{0.314} \\
\hline $\begin{array}{l}\text { Small Effect On } \\
\text { Patients Life }\end{array}$ & 9 & 22.5 & 4 & 10 & 10 & 25 & 9 & 22.5 & & & & \\
\hline $\begin{array}{l}\text { Moderate Effect } \\
\text { On Patients Life }\end{array}$ & 7 & 17.5 & 7 & 17.5 & 3 & 7.5 & 0 & 0 & & & & \\
\hline $\begin{array}{l}\text { Very large effect on } \\
\text { patient's life }\end{array}$ & 1 & 2.5 & 7 & 17.5 & 1 & 2.5 & 2 & 5.0 & & & & \\
\hline $\begin{array}{l}\text { Total DLQI score } \\
\bullet \quad \text { Mean } \pm \text { SD } \\
\bullet \quad \text { Median }\end{array}$ & \multicolumn{2}{|c|}{$\begin{array}{c}2.55 \pm 3.45 \\
0\end{array}$} & \multicolumn{2}{|c|}{$\begin{array}{c}4.15 \pm 5.50 \\
0\end{array}$} & \multicolumn{2}{|c|}{$\begin{array}{c}4.10 \pm 5.21 \\
2\end{array}$} & \multicolumn{2}{|c|}{$\begin{array}{c}1.43 \pm 3.51 \\
0\end{array}$} & ANOVA & 0.152 & 0.010 & 0.009 \\
\hline
\end{tabular}

P1: $1^{\text {st }}$ trimester vs controls P2: $2^{\text {nd }}$ trimester vs controls P3: 3rd trimester vs controls

There were significantly higher effects on patients' life and higher mean values of DLQI score among females with skin changes compared to those without $(\mathrm{p}<0.001)$. (Table 6).

Table (6): DLQI among the three trimesters pregnant females regarding skin changes.

\begin{tabular}{|c|c|c|c|c|c|c|c|c|c|c|c|c|c|c|c|}
\hline \multirow{3}{*}{ DLQLI } & \multicolumn{4}{|c|}{$1^{\text {st }}$ trimester pregnant } & \multicolumn{4}{|c|}{$\begin{array}{c}\text { 2nd trimester } \\
\text { pregnant }\end{array}$} & \multicolumn{4}{|c|}{$3^{\text {rd }}$ trimester pregnant } & \multirow{3}{*}{ P1 } & \multirow{3}{*}{ P2 } & \multirow{3}{*}{ P3 } \\
\hline & \multicolumn{2}{|c|}{$\begin{array}{l}\text { With skin } \\
\text { changes } \\
(\text { No =20) }\end{array}$} & \multicolumn{2}{|c|}{$\begin{array}{l}\text { Without } \\
\text { skin } \\
\text { changes } \\
(\text { No }=20)\end{array}$} & \multicolumn{2}{|c|}{\begin{tabular}{|l} 
With skin \\
changes \\
$($ No =20)
\end{tabular}} & \multicolumn{2}{|c|}{$\begin{array}{l}\text { Without } \\
\text { skin } \\
\text { changes } \\
(\text { No }=20)\end{array}$} & \multicolumn{2}{|c|}{$\begin{array}{l}\text { With skin } \\
\text { changes } \\
(\text { No }=20)\end{array}$} & \multicolumn{2}{|c|}{$\begin{array}{l}\text { Without } \\
\text { skin } \\
\text { changes } \\
(\text { No }=20)\end{array}$} & & & \\
\hline & No & $\%$ & No & $\%$ & No & $\%$ & No & $\%$ & No & $\%$ & No & $\%$ & & & \\
\hline $\begin{array}{l}\text { No effect at all } \\
\text { on patient's life }\end{array}$ & 4 & 20 & 19 & 95 & 4 & 20 & 18 & 90 & 0 & 0 & 17 & 85 & \multirow{4}{*}{$<0.001$} & \multirow{4}{*}{$<0.001$} & \multirow{4}{*}{$<0.001$} \\
\hline $\begin{array}{l}\text { Small Effect } \\
\text { On Patients } \\
\text { Life }\end{array}$ & 9 & 45 & 0 & 0 & 3 & 15 & 1 & 5 & 11 & 55 & 2 & 10 & & & \\
\hline $\begin{array}{l}\text { Moderate } \\
\text { Effect On } \\
\text { Patients Life }\end{array}$ & 6 & 30 & 1 & 5 & 6 & 30 & 1 & 5 & 4 & 20 & 1 & 5 & & & \\
\hline $\begin{array}{l}\text { Very large } \\
\text { effect on } \\
\text { patient's life }\end{array}$ & 1 & 5 & 0 & 0 & 7 & 35 & 0 & 0 & 5 & 25 & 0 & 0 & & & \\
\hline $\begin{array}{l}\text { Total DLQI } \\
\text { score } \\
\text { Mean } \pm \text { SD } \\
\text { Median }\end{array}$ & \multicolumn{2}{|c|}{$\begin{array}{c}4.50 \pm 3.38 \\
4\end{array}$} & \multicolumn{2}{|c|}{$\begin{array}{c}0.60 \pm 2.23 \\
0\end{array}$} & \multicolumn{2}{|c|}{$\begin{array}{c}7.80 \pm 5.65 \\
9.0\end{array}$} & \multicolumn{2}{|c|}{$\begin{array}{c}0.50 \pm 1.47 \\
0\end{array}$} & \multicolumn{2}{|c|}{$\begin{array}{c}7.50 \pm 5.11 \\
5\end{array}$} & \multicolumn{2}{|c|}{$\begin{array}{c}0.70 \pm 2.27 \\
0\end{array}$} & $<0.001$ & $<0.001$ & $<0.001$ \\
\hline
\end{tabular}

P1: females with skin changes vs females without skin changes in $1^{\text {st }}$ trimester group

P2: females with skin changes vs females without skin changes in $2^{\text {nd }}$ trimester group

P3: females with skin changes vs females without skin changes in $3^{\text {rd }}$ trimester group 


\section{DISCUSSION}

This study showed a significant difference between the three groups regarding the types of skin changes. Acne vulgaris was the most common in the $1^{\text {st }}$-trimester group, while generalized hyperpigmentation was common among the $2^{\text {nd }}$ and $3^{\text {rd }}$ trimester groups.

Two factors that make acne more prevalent in pregnancy are the progesterone hormone and changes in hydration. Progesterone causes increased sebum production and blockage of the pore openings which will lead to more acne ${ }^{(7)}$.

Similar to our result regarding pigmentation, the study conducted by Ahmad $\boldsymbol{e t}$ al. ${ }^{(8)}$, showed that pigmentary changes were the most common skin manifestations during pregnancy.

In our study, there was a significant relation regarding the skin changes and the number of previous pregnancies as skin changes increase especially in the $1^{\text {st }}$ trimester by an increased number of previous pregnancies $(\mathrm{p}=0.014)$. This is in agreement with Kannambal et al. (9), who reported that the multigravidae have significantly more vascular, pigmentary changes, and striae than primigravidae $(\mathrm{p}$ $<0.001)$.

Regarding sexual functions among the studied groups, there was a significant difference between the studied groups as regards frequency of intercourse $(p=0.041)$. Twice per week was the most common frequency in all groups as it accounts for $42.5 \%$ in control and $45 \%$ in $1^{\text {st }}$ trimester then declines to $37.5 \%$ in $2^{\text {nd }}$ trimester and rises to the highest level (55\%) in $3^{\text {rd }}$ trimester.

In a meta-analysis by Hasani et al. ${ }^{(10)}$, the mean of vaginal intercourse frequency was twice per week before pregnancy to be decreased during the first trimester of pregnancy to be once per week, while it increased in the second trimester to be twice per week and reduced again in the third trimester to be once or twice per month.

In this study, there were significant differences between the studied groups regarding the total FSDI, desire, lubrication, orgasm, and pain $(p<0.001$, $\mathrm{p}<0.001, \mathrm{p}=0.013, \mathrm{p}=0.010, \mathrm{p}<0.001$ respectively). Also, showed a significant difference regarding arousal score only between $2^{\text {nd }}$ trimester and control being the lower in $2^{\text {nd }}$ trimester $(\mathrm{p}=0.020)$. Moreover, it showed that the second-and third-trimester pregnant females groups suffer more from painful intercourse compared with the control group ( $22=0.009$ and $\mathrm{p} 3=0.015$, respectively)

Similar to our results, Aslan et al. (11), and Ahmed et al. ${ }^{(12)}$, showed significant differences between studied groups regarding the total FSDI and lubrication scores $(\mathrm{p}<0.000, \mathrm{p}=0.001$, resp. $)$ and $(p=0.001, p<0.000$ resp) respectively, where the highest mean level was in the prepregnancy period but the lowest was in the $3^{\text {rd }}$-trimester group. Also showed significant difference regarding the desired score between preconception and pregnant groups $(p<0.000$, $\mathrm{p}=0.001$, resp) but the score was highest in $1^{\text {st }}$ trimester and lowest in $3^{\text {rd }}$. Moreover, they showed a significant difference regarding arousal score between $2^{\text {nd }}$ trimester and pre-pregnant groups $(\mathrm{p}<0.000, \mathrm{p}=0.001$, resp).

They also showed significant differences regarding pain scores between the $2^{\text {nd }}$ and $3^{\text {rd }}$ trimester groups and the prepregnancy group $(\mathrm{p}<0.000$, $\mathrm{p}=0.001$, resp).

Similarly, Corbacioglu et al. (13), also demonstrated a significant decrease in orgasm score with the progression of pregnancy, the highest being in the $1^{\text {st }}$ and the lowest in the $3^{\text {rd }}$ trimester $(\mathrm{p}<0.05)$.

While Khalesi et al. ${ }^{(14)}$, reported a variable reduction of mean FSDI score between the three trimesters: 23.8 in the $2^{\text {nd }}$ trimester, 22.6 in the $1^{\text {st }}$ trimester, and 17.5 in the $3^{\text {rd }}$ trimester.

Hanafy et al. ${ }^{(15)}$, showed decreased sexual desire in the first trimesters, then it becomes variable in the second trimester and decreases at the end of the third trimester $(3.5 \pm 1.2,3.7 \pm 1.2$, and $3.4 \pm 1.1$, resp.).

In fact, with the progress of pregnancy, sexual desire, number of intercourses, number of orgasms, and sexual satisfaction decrease in most women. This is explained by fatigue, nausea, and vomiting during the 1st trimester, painful intercourses due to the anatomical changes as well as hypertrophy of pelvic viscera, and changes in women's mental images during the $3^{\text {rd }}$ trimester. Also, the mother's stress of being seen by the fetus, especially after the beginning of the fetal movements, can lead to a decrease in her sexual excitement ${ }^{(\mathbf{1 6})}$.

There was no significant difference between females with skin changes compared to those without regarding their sexual function among the three trimesters groups ( $p>0.05$ ). This is contrary to Kazemi et al. (2), who reported that unpleasant skin changes including acne, melasma, and striae made pregnant women suffer from body image disorder, leading to their decreased self-esteem and feeling ugly, affecting their sexual response. The difference in results might be due to different sample sizes, group characteristics, and cultures.

On the other hand, regarding DLQI total score, there was a significant difference between both the second and third trimesters and control ( $22=0.010$ and p3 $=0.009$, respectively). 
Some of the pregnant women suffered from body image disorder caused by pregnancy, leading to their decreased self-esteem. Moreover, unpleasant skin changes including acne, chloasma or melasma, and striae were among the reasons mentioned and some of the participants had a feeling of being ugly due to these body changes, affecting their QOL ${ }^{(2)}$.

In Szczęch et al. ${ }^{(17)}, 78 \%$ of pregnant females with pruritus had impaired quality of life and a significant correlation was noted between DLQI scoring and pruritus intensity

In our study, pregnant females with skin changes had significantly reduced quality of life compared to those without $(\mathrm{p}<0.001)$. Similar to our results, Ciechanowicz et al. ${ }^{(18)}$, reported about $43.67 \%$ of women with dermatological problems during pregnancy showed significant deterioration in their wellbeing. Furthermore, Uyanikoglu and Aksoy ${ }^{(19)}$, in their study, showed a slight but statistically insignificant reduced quality of life in pregnant females with melasma $(\mathrm{P}>0.05)$.

\section{CONCLUSION}

Pregnancy negatively affecting the female sexual function and skin changes during pregnancy not affecting it. Pregnancy and pregnancy-related skin changes significantly affect the quality of life of pregnant females.

\section{REFERENCES}

1. Hassan I, Bashir S, Taing $S$ (2015): A clinical study of the skin changes in pregnancy in Kashmir valley of north India: A hospital-based study. Indian Journal of Dermatology, 60(1):28-29.

2. Kazemi F, Nahidi F, Kariman N (2017): Disorders affecting the quality of life during pregnancy: A qualitative study. Journal of Clinical and Diagnostic Research, 11(4):6-7.

3. Förger F, Oestensen M, Schumacher A et al. (2005): Impact of pregnancy on health-related quality of life evaluated prospectively in pregnant women with rheumatic diseases by the SF-36 health survey. Annals of the Rheumatic Diseases, 64(10):1494-9.

4. Calou C, Pinheiro A, Castro R et al. (2014): Healthrelated quality of life of pregnant women and associated factors: An integrative review. Health, 2(2):67-69.

5. Anis T, Gheit S, Saied H et al. (2011): Arabic translation of Female Sexual Function Index and validation in an Egyptian population. The Journal of Sexual Medicine, 8(12):3370-8.

6. Basra M, Fenech R, Gatt $\mathrm{R}$ et al. (2008): The Dermatology Life Quality Index 1994-2007: a comprehensive review of validation data and clinical results. British Journal of Dermatology, 159(5):9971035.

7. Bayerl C (2013): Acne therapy in pregnancy. Hautarzt., 64(4):269-73.

8. Ahmad H, El Sharkawy R, Rasheed S (2018): Cutaneous Changes During Pregnancy. Sohag Medical Journal, 22(3):381-9.

9. Kannambal K, Tharini G (2017): A screening study on dermatoses in pregnancy. Journal of Clinical and Diagnostic Research, 11(5): 1-3.

10. Hasani M, Keramat A, Maasoumi R et al. (2019): The frequency of vaginal intercourse during pregnancy: a systematic and meta-analysis study. International $\mathbf{J}$ Women's Health Reproduc Sci., 7(2):1-9.

11. Aslan G, Aslan D, Kizllyar A et al. (2005): A prospective analysis of sexual functions during pregnancy. International Journal of Impotence Research, 17(2):154-7.

12. Ahmed M, Madny E, Sayed Ahmed W (2014): Prevalence of female sexual dysfunction during pregnancy among Egyptian women. J Obstet Gynaecol Res., 40(4):1023-1029.

13. Corbacioglu Esmer A, Akca A, Akbayir O et al. (2013): Female sexual function and associated factors during pregnancy. Journal of Obstetrics and Gynaecology Research, 39(6):1165-72.

14. Khalesi Z, Bokaie M, Attari S (2018): Effect of pregnancy on sexual function of couples. African Health Sciences, 18(2):227-34.

15. Hanafy S, Srour N, Mostafa T (2014): Female sexual dysfunction across the three pregnancy trimesters: an Egyptian study. Sexual Health, 11(3):240-3.

16. Jamali S, Mosalanejad L (2013): Sexual dysfnction in Iranian pregnant women. Iranian Journal of Reproductive Medicine, 11(6):479-480.

17. Szczęch J, Wiatrowski A, Hirnle L et al. (2017): Prevalence and relevance of pruritus in pregnancy. BioMed Research International, 2(2):47-50.

18. Ciechanowicz P, Sikora M, Taradaj K et al. (2018): Skin changes during pregnancy. Is that an important issue for pregnant women? Ginekologia Polska., 89(8):450-3.

19. Uyanikoglu H, Aksoy M (2017): Quality of life in patients with melasma in Turkish women. Dermatology Reports, 9(2):54-60. 